\title{
A blend of traditional visual symbols in BIPV application: any prospects?
}

AWUKU, S.A., BENNADJI, A., MUHAMMAD-SUKKI, F. and SELLAMI, N. 


\title{
ACADEMIA $\mid$ Letters
}

\section{A blend of Traditional Visual Symbols in BIPV application; any Prospects?}

\author{
Samuel Amo Awuku, Robert Gordon University, School of Engineering \\ Amar Bennadji, Robert Gordon University, Scott Sutherland School of Architecture \\ and Built Environment \\ Firdaus Muhammad-Sukki, Edinburgh Napier University, School of Engineering \\ and the Built Environment \\ Nazmi Sellami, Edinburgh Napier University, School of Engineering and the Built \\ Environment
}

\begin{abstract}
Traditional Visual symbols are of great relevance, especially within the architectural space. Buildings may adopt symbols to depict culture, iconicity or identity. The quest to meet sustainable building targets and avert the looming effects of climate change has brought about renewable options such as Building Integrated Photovoltaics (BIPV), which is the use of solar PVs to replace conventional building materials such that they form part of the building envelope. This paper discusses the nexus between traditional visual symbols and BIPV application. A hypothetical question is posed; will incorporating visual symbols in BIPV design influence its adoption? Although a detailed evidence-based result is not produced in this paper, architectural visualisation is used to depict the outlook of traditional Ghanaian symbols in BIPV application, while further studies is being conducted into the prospects of these symbols in BIPVs design using Traditional Ghanaian "Adinkra" symbols as case study.
\end{abstract}

Academia Letters, November 2021

(C)2021 by the authors - Open Access — Distributed under CC BY 4.0

Corresponding Author: Samuel Amo Awuku, nanaawuku021@gmail.com

Citation: Awuku, S.A., Bennadji, A., Muhammad-Sukki, F., Sellami, N. (2021). A blend of Traditional Visual Symbols in BIPV application; any Prospects? Academia Letters, Article 4029.

https://doi .org/10.20935/AL4029. 


\section{Introduction}

Visual symbols are of great relevance and a major mark of exoticism especially in architecture. Throughout history, buildings have been made to demonstrate, convey or depict particular messages and ideas, either as a form of representation, identity or iconicity. Sometimes, buildings are used to demonstrate the culture of a particular people. For short, symbols in buildings are of cognitive and communicative relevance [1]. A considerable attempt has been made to incorporate traditional symbols in modern architecture. The idea is to develop buildings that are of traditional and cultural relevance. The post-modern architecture draws inspiration from historic symbols which connects to the people [2]. Symbolism can be said to have a close relationship with the economic and cultural growth of a society [3]. This paper highlights a brief perspective on traditional symbols in BIPV application. Although an evidence-based result is not produced in this short paper, it provides a solid ground for future research work.

\section{Visual Symbols in Buildings and the new BIPV}

These days, buildings are constructed with sustainability, efficiency and aesthetics forming their backbone. Buildings have moved from mere functionality to become sustainable and energy efficient. Part of this can be attributed to the recent chain-reactions of climate change on the ecosystem, aquatic species and human life. This has necessitated a swift approach to reduce carbon emissions and warranted the explorations of cleaner energy options such as renewables in place of cruddy sources such as fossil fuel. The idea is to rely on cleaner sources for global energy needs. Renewables such as solar energy has seen massive transformation in technology as the rate of adoption increases. Developers have come up with various modern solar systems in both Photovoltaics (PV) and Concentrated Solar thermal Power (CSP) [5]. The growth in PV technology has seen the inception of efficient and aesthetically pleasant solar application such as Building Integrated Photovoltaics (BIPV). BIPV is the use of solar photovoltaics to replace conventional building components such that they form part of the building envelope after construction [6]. BIPVs provides an aesthetically pleasant application of PV in buildings, while providing clean energy and offsetting the cost of original building materials [7]. A classic example is shown in figure 1 below. The advantages are numerous, yet its penetration into the PV market has been relatively slow [8], [9].

Corresponding Author: Samuel Amo Awuku, nanaawuku021@gmail.com

Citation: Awuku, S.A., Bennadji, A., Muhammad-Sukki, F., Sellami, N. (2021). A blend of Traditional Visual Symbols in BIPV application; any Prospects? Academia Letters, Article 4029. https://doi .org/10.20935/AL4029. 


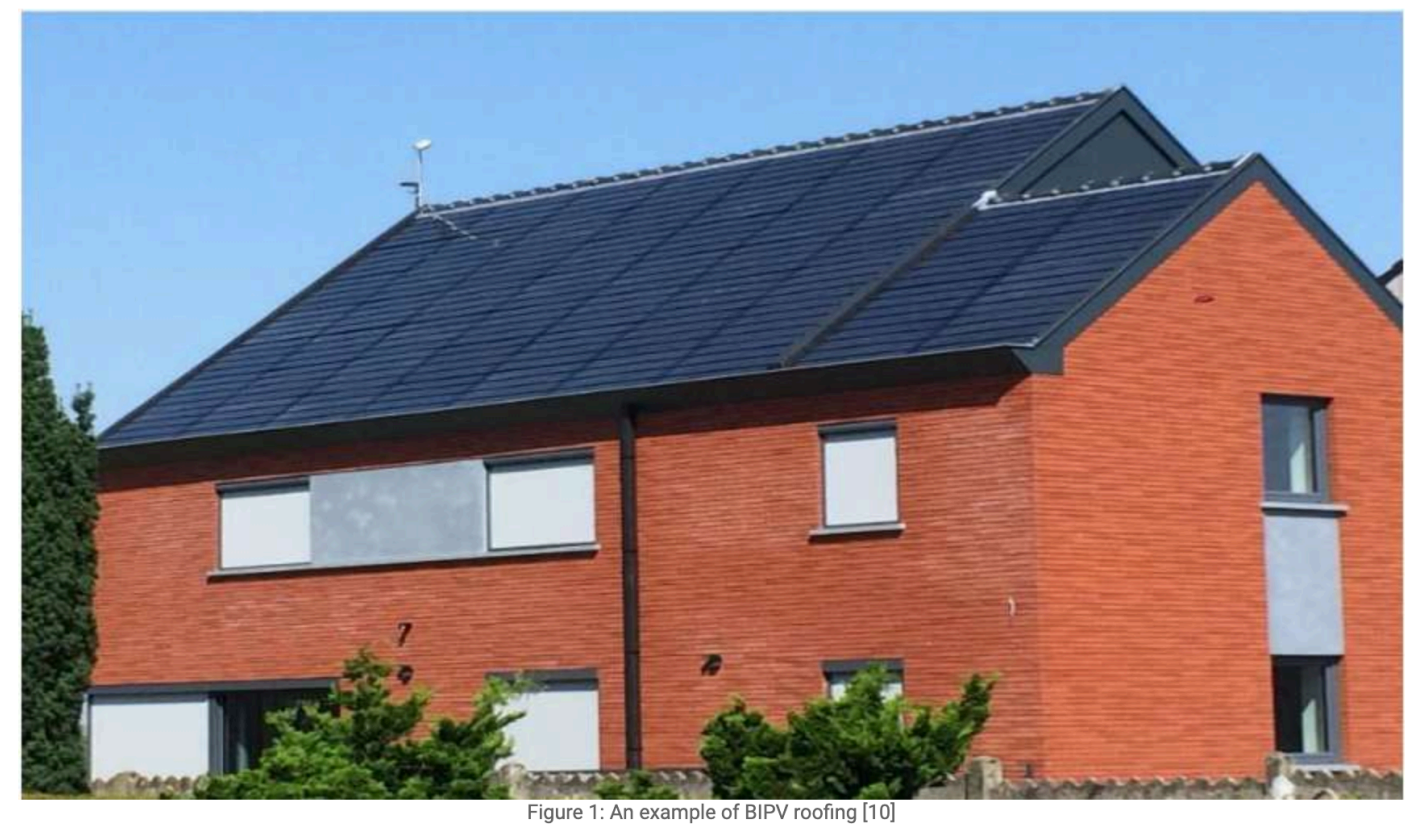

As the quest for sustainable buildings increases, especially after policies such as "Net Zero Energy Buildings" (NZEB) [11], BIPV promises to be a viable energy source that easily blends with modern architectural materials. The traditions and culture of a group of people cannot be substituted, hence the incorporation of significant and meaningful symbols in modern architectural materials could go a long way to encourage its adoption. For instance, if cladding, façade, or roofing materials should incorporate traditional symbols, there is a tendency that they may appeal to a certain calibre of people. It is evident that, the post-modern era has an interesting connection with antiquity. In other words, people are attracted to indigenous things, and may want to readopt them even in this modern era. Traditional symbols have lived for centuries, hence have great bearing on the ethos of a cultural group. Adopting these symbols in modern architecture has been championed by many researchers and designers [12], [3], [13].

The conscious application of traditional visual symbols in BIPV design promises to be a booster for PV adoption. BIPV replaces conventional building materials, hence there is room to manipulate and develop them to mimic original building materials without compromising on their energy producing ability. Traditional visual symbols have been known to boost product adoption in especially cultural areas. Many people naturally remain patriotic and hold their

Corresponding Author: Samuel Amo Awuku, nanaawuku021@gmail.com

Citation: Awuku, S.A., Bennadji, A., Muhammad-Sukki, F., Sellami, N. (2021). A blend of Traditional Visual Symbols in BIPV application; any Prospects? Academia Letters, Article 4029. https://doi.org/10.20935/AL4029. 
heritage in high esteem, hence are quick to identify with symbols and icons. Once BIPV's promises to replace conventional building materials such as roof and facades, the possibility of traditional display promises to be an attractive feature that could potentially draw adopters.

The BIPV technology has grown exponentially, especially in recent times as the quest to migrate existing and new buildings to net zero by 2050 increases [14]. From colour, shape, texture etc., BIPV materials have evolved considerably with major hit backs such as efficiency and other technical limitations being resolved. These days, BIPV materials appear in different colours and shapes, yet remain efficient to a large extent [15]. An introduction of traditional visual symbolism will therefore be a variation of a "tried and tested" application and does not promise to cause a wide difference in cost or perhaps compromise the efficiency of the BIPV material. Although this remains a very novel area yet to be explored, the prospects seem numerous, considering the fundamental nexus between traditional symbolism and modern architecture.

\section{The Ghanaian Adinkra Case Study}

Adinkra symbols are of great traditional and cultural relevance to Ghanaians. These symbols have been replicated on various substrates, especially on clothes and monumental buildings [16]. Considering the fact that BIPVs are replacing conventional building materials, a Ghanaian Adinkra symbol known as "Gye Nyame" (Only God), which signifies the supremacy of God has been incorporated in the design of BIPV for a selected local building in Ghana. Figure 2 below shows demonstrates this.

Corresponding Author: Samuel Amo Awuku, nanaawuku021@gmail.com

Citation: Awuku, S.A., Bennadji, A., Muhammad-Sukki, F., Sellami, N. (2021). A blend of Traditional Visual Symbols in BIPV application; any Prospects? Academia Letters, Article 4029. 


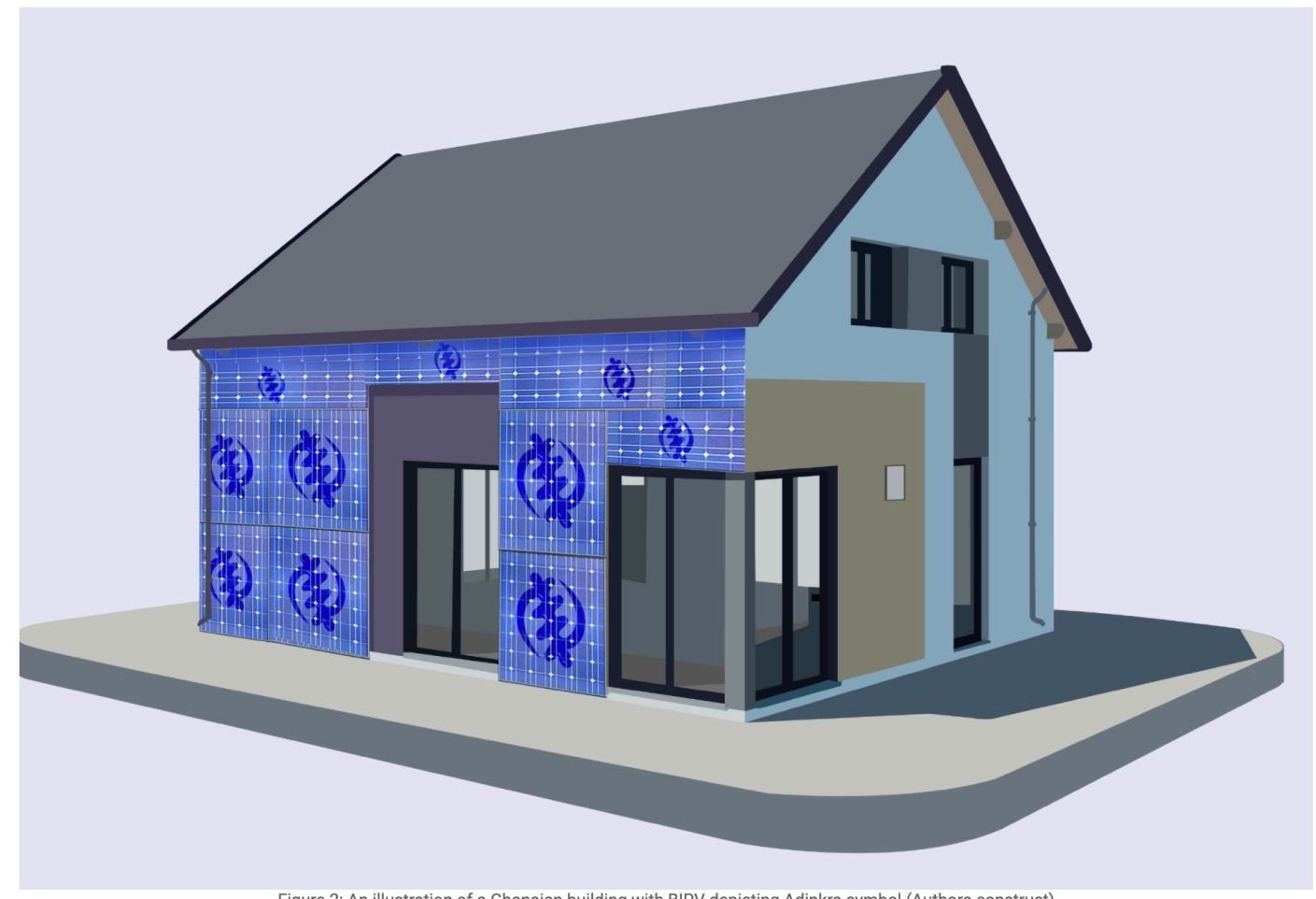

Figure 2: An illustration of a Ghanaian building with BIPV depicting Adinkra symbol (Authors construct)

\section{Conclusion}

Symbols have great bearing with modern architecture, hence the conscious approach to blend these two. Considering the fact that BIPVs have come to replace conventional building materials aside meeting the energy needs of a building, incorporating traditional visual symbols could go a long way to put BIPVs up as an "environmentally friendly energy solution that blends modern architecture with tradition and culture".

Corresponding Author: Samuel Amo Awuku, nanaawuku021@gmail.com

Citation: Awuku, S.A., Bennadji, A., Muhammad-Sukki, F., Sellami, N. (2021). A blend of Traditional Visual Symbols in BIPV application; any Prospects? Academia Letters, Article 4029. https://doi.org/10.20935/AL4029. 


\section{References}

[1] N. Shi, "The Application of Chinese Traditional Cultural Symbols in Architectural Design,” 2018, doi: 10.25236/iseah.2018.042.

[2] E. Haddad, "Charles Jencks and the historiography of Post-Modernism," Taylor Fr., vol. 14, no. 4, pp. 493-510, Aug. 2009, doi: 10.1080/13602360902867434.

[3] M. Mankus, "Manifestations of symbolism in architecture of postmodernism," J. Archit. Urban., vol. 38, no. 4, pp. 274-282, Jan. 2014, doi: 10.3846/20297955.2014.998853.

[4] U. D. of Energy, "How Does Solar Work? | Department of Energy," 2021. https://www. energy.gov/eere/solar/how-does-solar-work (accessed Oct. 27, 2021).

[5] C. Peng, Y. Huang, and Z. Wu, "Building-integrated photovoltaics (BIPV) in architectural design in China," Energy Build., vol. 43, no. 12, pp. 3592-3598, 2011, doi: https://doi. org/10.1016/j.enbuild.2011.09.032.

[6] B. P. Jelle, C. Breivik, and H. Drolsum Røkenes, "Building integrated photovoltaic products: A state-of-the-art review and future research opportunities," Sol. Energy Mater. Sol. Cells, vol. 100, pp. 69-96, May 2012, doi: 10.1016/j.solmat.2011.12.016.

[7] R. A. Agathokleous and S. A. Kalogirou, "Status, barriers and perspectives of building integrated photovoltaic systems," Energy, vol. 191, p. 116471, Jan. 2020, doi: 10.1016/J.ENERGY.2019.116471.

[8] J. Koinegg, T. Brudermann, A. Posch, and M. Mrotzek, "It would be a shame if we did not take advantage of the spirit of the times ': An analysis of prospects and barriers of Building integrated photovoltaics," GAIA, vol. 22, no. 1, pp. 39-45, 2013, doi: 10.14512/GAIA.22.1.11.

[9] E. Africa, "Design engineering and construction of BIPV," 2017. https://www.esi-africa. com/magazine-article/design-engineering-and-construction-of-bipv/ (accessed Jul. 21, 2021).

[10] “NZEB | Energy.” https://ec.europa.eu/energy/content/nzeb-24_en (accessed Oct. 27, 2021).

[11] J. Alford, "Modern architecture and the symbolism of creative process," 1955 College Art J., vol. 14, no. 2, pp. 102-123, 1955, doi: https://doi.org/10.2307/773021.

[12] V. Khaidukov and O. Sleptsov, "Architectural symbolism in tradition and modernity,"

Academia Letters, November 2021

C 2021 by the authors - Open Access — Distributed under CC BY 4.0

Corresponding Author: Samuel Amo Awuku, nanaawuku021@gmail.com

Citation: Awuku, S.A., Bennadji, A., Muhammad-Sukki, F., Sellami, N. (2021). A blend of Traditional Visual Symbols in BIPV application; any Prospects? Academia Letters, Article 4029.

https://doi .org/10.20935/AL4029. 
2020, doi: 10.1088/1757-899X/913/3/032024.

[13] “The Net Zero Carbon Buildings Commitment / World Green Building Council.” https:// www.worldgbc.org/thecommitment (accessed Nov. 03, 2021).

[14] G. Eder et al., "Coloured BIPV,” 2019, Accessed: Nov. 03, 2021. [Online]. Available: https://iea-pvps.org/wp-content/uploads/2020/01/IEA-PVPS_15_R07_Coloured_BIPV_report. pdf.

[15] J. Aboagyewaa-Ntiri, C. B. Campion, and A. K. Kemevor, "Extension of Knowledge on Ghanaian Adinkra Symbols in Relation to Maslow's Theory," 2018, Int. J. Netw. Commun. Res., vol. 5, no. 1, pp. 11-24, 2018, Accessed: Aug. 11, 2021. [Online]. Available: www.eajournals.org. 